\title{
Verifiability and Symptom Endorsement in Genuine, Exaggerated, and Malingered Pain
}

\author{
Y. E. Ali Akca ${ }^{1} \cdot$ Lotte Slootmaekers $^{1} \cdot$ Irena Boskovic ${ }^{1,2}$ (D) \\ Received: 25 February 2020 / Accepted: 13 April 2020 / Published online: 4 May 2020 \\ (C) The Author(s) 2020
}

\begin{abstract}
The current study has investigated whether pure malingering, in which reported symptoms are nonexistent, partial malingering, in which existent symptoms are exaggerated, and genuine symptoms could be differentiated by applying the verifiability approach (VA) and the Self-Report Symptom Inventory (SRSI). The logic behind the VA is that deceivers' statements contain more nonverifiable information, whereas truth tellers' accounts include more verifiable details. The SRSI taps into over-reporting by including a mix of genuine symptoms and implausible complaints (pseudosymptoms). We checked if participants $(N=167)$ allocated to one of three conditions (pure malingerers vs. exaggerators vs. truth tellers) can be differentiated in their pain symptom reports' (non)verifiability and symptom endorsement. Findings revealed that deceptive reports were lengthier than truthful statements. However, this difference was not produced by a discrepancy in non-verifiable details, but rather by a higher production of verifiable information among malingerers and exaggerators. Thus, contrary to previous findings, our results indicate that pain reports rich in verifiable information should raise doubt about their veracity. Further, truth tellers endorsed less symptoms of the SRSI than exaggerators, but not than pure malingerers. Pure malingerers and exaggerators did not differ in symptom endorsement. Thus, our findings revealed that when compared with truth tellers, exaggerators exhibited stronger overreporting tendencies than (pure) malingerers. However, due to inconsistent findings, further investigation of the efficacy of these methods in differentiation between exaggerated and malingered reports is required.
\end{abstract}

Keywords Exaggeration · Malingering $\cdot$ Verifiability $\cdot$ Endorsement $\cdot$ Symptom reports

Whether driven by an incentive or by an outcome of a decision (e.g., deceive or disclose; Rogers, 2018a), response bias taints the validity of clinical and forensic assessment. A response style could be positive, meaning that a person is intentionally or unintentionally (mis)presenting and under-reporting problems associated with their mental or physical health to portray themselves in a better light than may be objectively determined (e.g., faking good in order to obtain certain benefits, such as hospital release). However, a person can also exhibit a negative response style (i.e., faking bad), when intentionally or unintentionally presenting their state as being worse than it is (i.e., over-reporting) or when claiming to experience a

Electronic supplementary material The online version of this article (https://doi.org/10.1007/s12207-020-09375-w) contains supplementary material, which is available to authorized users.

Irena Boskovic

irena.boskovic@maastrichtuniversity.nl; boskovic@essb.eur.nl

Maastricht University, Maastricht, the Netherlands

2 Erasmus University Rotterdam, Rotterdam, the Netherlands condition they do not actually have (i.e., faking bad in order to obtain an incentive, such as disability compensation). Response styles are not limited to one specific domain of mental or medical health. Three domains in which response biases have been extensively researched are reports about mental disorders, cognitive abilities, and medical complaints/symptoms (Rogers, 2018a). People might show either positive or negative response styles for a variety of reasons. Some people might unintentionally do so due to a poor ability to inspect their inner experiences (e.g., alexithymia) or due to certain personality traits (e.g., fantasy proneness; Merckelbach, Boskovic, Pesy, Dalsklev, \& Lynn, 2017). However, in some cases, people consciously and deliberately exhibit a negative response style on symptom inventories in order to obtain some kind of external incentive (i.e., malingering; DSM-5, APA, 2013; Merten \& Merckelbach, 2013). External incentives may include financial gains (e.g., legal compensations), avoiding (criminal) responsibility, and obtaining drugs or medication but are not limited to these.

The most cited base rates for malingering in the existing literature are provided by Mittenberg, Patton, Canyock, and 
Condit (2002). Based on a survey involving 131 active practicing neuropsychologists in the USA and Canada, probable malingering was prevalent in 8 to $33 \%$ of the annual 33,531 neuropsychological examinations. However, recent review of base rate studies has suggested that the prevalence of malingering is $15 \pm 15 \%$ (Young, 2015). Moreover, Young (2015) stated that the highly varying base rates, ranging from 3 to $64 \%$, appear to be the result of multiple factors. These factors include the referral type (e.g., mild traumatic brain injury, psychiatric), criteria used to detect malingering, the assessment methods, and the definition of malingering.

A prominent categorization of malingering has been suggested by Resnick (1984), who upholds that three types of malingering can be distinguished as follows: (1) Pure malingering, (2) partial malingering, and (3) false imputation. Individuals who consciously and deliberately fabricate symptoms and/or disorders that do not exist for the purpose of obtaining an external incentive are categorized as pure malingerers. An individual is considered a partial malingerer when he or she exaggerates symptoms that are genuinely present. Further, individuals fall in the false imputation category when they attribute genuine symptoms to a cause different from the one their symptoms actually originate from. Important to note is that, for each category, volition and intentionality are key characteristics (Resnick, West, \& Wooley, 2018). Iverson (2006) indicated that such a taxonomy of malingering is important because practitioners involved in symptom validity assessment might embrace a simplistic understanding of the concept. That is, that the only type of malingering is pure malingering.

Interpreting exaggeration of symptoms on its own as malingering in the absence of corroborative evidence is an ethical concern (Iverson, 2006). Accordingly, Iverson (2007) postulated that exaggeration is an important characteristic of malingering, though it does not always indicate malingering. For example, malingering will always lead to exaggeration/overreporting and poor effort, but exaggeration/over-reporting and poor effort are not necessarily cues to malingering, as other factors might be underlying this response style. For instance, research has shown that individuals with certain personality traits, such as high levels of fantasy proneness (i.e., richness in imagination; Peace \& Masliuk, 2011; Merckelbach et al. 2017), as well as people who have difficulties in recognizing internal sensations, a trait known as alexithymia (see Peace \& Bouvier, 2008), often exhibit atypical trauma symptom reports. Individuals with iatrogenic symptoms might also be susceptible to inflation of symptom reports, especially in case of complaints without medical etiologies (Bender \& Matusewicz, 2013). Further, other psychological factors, such as catastrophizing (see Nicholson, 2000) or even perfectionism (Hadjistavropoulos, Dash, Hadjistavropoulos, \& Sullivan, 2007), could also influence the individual's response style. Education and reading levels of a person should also be considered in any type of symptom assessment (for recommendations see Merten et al. 2019), as well as the language (Harzing, 2006) and culture (Nijdam-Jones \& Rosenfeld, 2017) in which the assessment in conducted. Ignoring these, and numerous other factors (e.g., social influence; see McCracken, 2005), has a severe impact on the estimated prevalence of pure malingering, but also the efficiency of the detection tools researchers employ in symptom validity assessment (for more see Rogers, 2018a, 2018b).

One of the most commonly used methods to detect malingering is to employ the Symptom validity tests (SVTs). SVTs can be administered as stand-alone measures, or they can be imbedded in other instruments (see Nelson, Hoelzle, Sweet, Arbisi, \& Demakis, 2010). Stand-alone SVTs include performance validity tests (PVTs) and the self-report symptom validity tests (SRSVTs). Whereas the first one assesses underperformance (i.e., poor effort) on cognitive tests, the latter one assesses over-endorsement of symptoms (Merten \& Merckelbach, 2013; Merten, Merckelbach, Giger, \& Stevens, 2016). The idea behind the SRSVTs is that individuals who exaggerate genuine or fabricate nonexistent symptoms are inclined to over-report their poor health claims (Boskovic, Merckelbach, Merten, Hope, \& Jelicic, 2019). Thus, SRSVTs heavily rely on bizarre or atypical symptoms that are unlikely to be experienced by genuine patients. Thus, if a person claims to experience those complaints, it is likely that this person is over-reporting his or her symptoms.

Recently, a new SRSVT was developed by Merten et al. (2016). The Self-Report Symptom Inventory (SRSI) consists of 107 true-false items divided into two main scales, the genuine symptoms and the pseudosymptoms scales (Merten et al., 2016). Each main scale consists of five subscales assessing either plausible or implausible symptoms of clinical, physiological, and cognitive domains. Hence, the SRSI has a wide utility in civil arena (e.g., compensation litigations; Merten et al., 2016) and has been adapted to several languages (e.g., Dutch, English, French; Giger \& Merten, 2019). Research so far showed that the SRSI is a promising method of detecting malingering of psychological phenomenon, with an overall detection rate of approximately $80 \%$ (Merten et al., 2016; van Helvoort, Merckelbach, \& Merten, 2019). However, using a previously recommended cutoff score, in the domain of pain-related issues, the SRSI performs below chance level (50\%; Boskovic et al., 2019).

Pain specifically presents a challenge for practitioners to assess as it relies on patients' self-report, and even implementing the SRVTs does not seem to significantly improve the detection of fabricated pain (for an overview, see Nicholson \& Martelli, 2007). Thus, its detection requires testing new approaches. For instance, Boskovic, Bogaard, Merckelbach, Vrij, and Hope (2017) checked the utility of a new verbal credibility assessment method, the Verifiability Approach (VA; Nahari, Vrij, \& Fisher, 2014), in detection of 
malingered physical complaints. The rationale behind the VA is that deceivers' accounts include more details that cannot be verified (i.e., non-verifiable details; e.g., "sharp," "burning pain"), whereas truth tellers exhibit more information that can be verified (i.e., verifiable details; e.g., "prescription"; "doctor appointment") in their statements. Applying the VA to detect malingerers, Boskovic et al. (2017) found that subjects instructed to simulate malingering or to fabricate symptoms provided significantly longer and non-verifiable reports than truth tellers. Hence, researchers concluded that the VA might aid the detection of malingering. However, the VA's applicability is limited, as it was shown that a majority of individuals claiming pain symptoms rarely include verifiable information in their reports if not asked to do so. Yet, once asked, subjects who fabricated symptoms exhibited a tendency to bluff (i.e., provide false verifiable details; Boskovic et al., 2017).

However, despite ongoing research on various methods for detecting malingering, no research thus far has found a method that could reliably distinguish different categories of symptom fabrication. For instance, it is still unclear how to differentiate pure malingering or partial malingering (i.e., exaggeration) from genuine pain symptoms (see Nicholson \& Martelli, 2007). The potential experimental evidence for this differentiation could have positive outcomes for the medico-legal context, especially practitioners' decision-making concerning the type of treatment a patient should receive could profit from a better understanding of the differences. A wrong decision can hurt not only patients with genuine symptoms but also partial malingerers who, if labeled as pure malingerers, are also at risk of being excluded from the needed care. Thus, a central question of this study was whether people reporting genuine pain-related symptoms (truth tellers) exhibit similar symptom endorsement (SRSI) and accounts' (non)verifiability (VA) as people who exaggerate (exaggerators) or fully fabricate such complaints (malingerers). We opted for a student sample, as previous studies confirmed a high prevalence of chronic pain-related symptoms in this population (see Hadjistavropoulos et al., 2007). This study hypothesized that, using the SRSI, truth tellers would endorse the lowest number of symptoms, followed by exaggerators who would endorse significantly more symptoms than truth tellers, but fewer symptoms than malingerers, for whom we anticipated the highest symptom endorsement. This direction of differences was expected to be found on both the genuine symptoms and pseudosymptoms scales. Further, we anticipated that the amount of (non)verifiable information would be similar among of the accounts of exaggerators and truth tellers, as they are based on genuinely existing symptoms, whereas malingerers would provide significantly more nonverifiable information.

\section{Methods}

\section{Participants}

Following a $G^{*}$ Power analysis, the suggested minimum sample size was 159 participants. The suggestion was based on $F$ set at .25 , alpha set at .05 , beta set at .80 , and number of groups at three.

Initially, 211 university students were recruited. However, of the recruited sample, 44 participants were excluded from the analysis due to either not completing the study $(n=38)$ or not satisfying the eligibility criteria $(n=6$; see below). The final sample consisted of 167 participants, most of whom were female (79\%), one participant preferred not to specify their gender. The mean age was $21.89(S D=3.39)$ and ranged from 18 to 35 years. The top three nationalities that participants identified with were as follows: (1) German ( $n=78$; 46.7\%), (2) Dutch ( $n=39 ; 23.4 \%)$, and (3) Italian ( $n=7$; 4.2\%; see Appendix 2 Supplementary material). According to the research outline (see "Procedure" section), participants were assigned to one of the three conditions: truth tellers $\left(n_{1}=64\right)$, partial malingerers (i.e., exaggerators; $\left.n_{2}=63\right)$, and pure malingerers (i.e., malingerers; $n_{3}=40$ ). Participants were allowed to choose whether they completed the tasks in either English or Dutch. As a result, 133 participants (79.6\%) preferred to respond in English, the remaining 20.4\% responded in Dutch. Participants who responded in English graded their proficiency on a five-point scale ( $1=$ "Extremely bad"; $5=$ "Extremely good"). The mean value for the sample $(N=133)$ was $M=4.46(S D=.58)$, indicating a very high English proficiency of participants.

This study was reviewed and approved by the Ethical Review Committee Psychology and Neuroscience of Maastricht University (Master_204_06_02_2019).

\section{Materials and Measures}

The Self-Report Symptom Inventory The SRSI consists of 107 true-false items divided into two main scales, the genuine symptoms and pseudosymptoms scales, in order to examine symptom over-endorsement (Merten et al., 2016). Items are related to either genuine symptoms (50 items) or pseudosymptoms (50 items). Each main scale contains five subscales focusing on various domain symptoms. The subscale domains of genuine symptoms are cognitive, depression, pain, nonspecific somatic complaints, and PTSD/anxiety. The domains being addressed on the pseudosymptoms scale are cognitive/memory, neurological (motoric) problems, neurological (sensory) problems, pain, and anxiety/depression (incl. PTSD). Each subscale has 10 items. Scores that can be obtained range from 0 to 50 for each main scale and 0 to 10 for each subscale. Over-endorsement is assessed by the 
pseudosymptoms scale. A standard cutoff score of more than nine pseudosymptoms is recommended for diagnostic purposes, whereas for screening purposes, a score of $>6$ on the pseudosymptoms scale should be asserted (Merten et al., 2016). In addition to the main scale items, the SRSI has seven items that tap into a priori cooperativeness and consistency. The first two items address understanding the instructions, whereas the remaining items tap into good health and mental abilities. Participants who over-report symptoms are expected to endorse a lower number of these items than honest subjects (see Boskovic, Merckelbach, et al., 2019; Merten et al., 2016). Our truth tellers $(M=2.87, S D=1.67)$ endorsed a significantly higher number of these items than both exaggerators $(M=$ 1.57, $S D=1.92)$ and malingerers $(M=1.49, S D=1.75 ; F(2$, $166)=11.64, p<.001, \eta_{p}{ }^{2}=.12$ ). In this study, two additional items (e.g., to this item respond with "False") were included to the SRSI in order to check for random responding. These items were introduced randomly in the scale. One participant responded incorrectly to one of the checks. ${ }^{1}$ The internal consistency estimate of reliability resulted in Cronbach's $\alpha$ of .93 for the genuine symptom scale, Cronbach's $\alpha$ of .92 for the pseudosymptom scale, and Cronbach's $\alpha$ of .96 for the total scale.

Verifiability Approach and Coding The logic behind the VA is that deceivers will provide more non-verifiable information, whereas truth tellers exhibit details that could be checked (i.e., verifiable). In this study, details in written statements of participants were coded as either verifiable $(\mathrm{V})$ or non-verifiable (NV). The criteria for details to be coded as verifiable were as follows: (a) the details are documented, and thus checkable (e.g., appointment with a doctor, prescriptions); (b) details involve an action carried out with (an)other identified person(s) rather than alone or with a non-traceable stranger (e.g., going to the doctor with a parent); (c) details pertain to something that was witnessed by (an) other identified person(s) (e.g., calling a doctor when someone else is present); (d) details are reported as being recorded (e.g., on CCTV; e.g., entering a pharmacy); (e) the use of technology (e.g., phone, tablet, or computer; e.g., googling symptoms, making a doctor's appointment online; Nahari \& Vrij, 2014); or (f) details which can potentially be checked by blood analysis and/or medical tests (e.g., taking specific pills; Boskovic, Gallardo, Vrij, Hope, \& Merckelbach, 2018). Details that did not meet the criteria above were coded as non-verifiable. Two coders, both blind to the conditions, carried out the coding. A primary coder was assigned to code all statements, whereas a randomly selected sample of approximately $20 \%(n=29)$ of the statements was coded by the second coder. An inter-rater reliability

\footnotetext{
${ }^{1}$ This participant provided a moderate symptom report and a statement. The data were analyzed without this participant, and the results did not change; thus, participant's data were kept in the data set.
}

analysis between coders was performed by means of calculating the interclass correlation coefficients (ICC). Based on a two-way random effects model, mean-rating, and consistency, the analysis resulted in a "good" to "excellent" ICC for both V and NV details (Koo \& Li, 2016). To be specific, for V details $\mathrm{ICC}=.89,95 \%$ CI $(.76-.95)$ and for NV details $\mathrm{ICC}=.88$, 95\% CI (.74-.94), respectively.

Per participant, verifiable and non-verifiable details were added in order to calculate absolute numbers for both. The proportion of verifiable details was calculated using the formula: verifiable details/(Verifiable details + Non-verifiable details).

\section{Procedure}

In this project, we followed a between-groups design using a simulation paradigm, meaning that participants received instructions in the form of vignettes to simulate a certain condition. Both the SRSI and the VA have been used in simulation design studies (see Boskovic et al., 2017; Boskovic, Dibbets, et al., 2019; Boskovic, Merckelbach, et al., 2019).

Participants were recruited through Maastricht University Research Participation System (SONA). After signing up for the study via SONA, participants received a link to Qualtrics, which was the medium used to gather all the data. Participants were provided with an option to either complete the study items in Dutch or English. Subsequently, all participants were asked whether they have experienced pain symptoms for the majority of time during the last week including the day they partook in the study. The list of symptoms participants could choose from contained the most encountered symptoms in general population (e.g., back or neck pain, joint pain or stiffness, and menstruation pain; see Petrie, Faasse, Crichton, \& Grey, 2014) and an option stating, "no symptoms". The list of selected symptoms is presented in Table 1. Additionally, participants also had the option to add symptoms to the list. Participants who reported experiencing one or more symptoms were asked about the intensity of those symptoms. Any participant who scored equal to or above $4(1=$ "Very low" to $5=$ "Extremely strong") was excluded from the study. We could not ask participants who scored that high to exaggerate their complaints, neither could we have expected participants in such discomfort to provide the detailed information that we needed. The remaining symptomatic participants were then randomly allocated to the truth telling or exaggeration condition. Participants who stated not experiencing any symptoms were assigned to the pure malingering condition (i.e., malingerers group). These asymptomatic participants were then asked to choose a symptom they would fabricate from the same list previously presented to them. Following, all participants received a vignette with instructions. Participants in the truth telling condition needed to respond honestly; 
Table 1 List of symptoms provided in the study and their frequencies

\begin{tabular}{|c|c|c|c|c|c|c|}
\hline \multirow[t]{2}{*}{ Physical symptoms } & \multicolumn{6}{|l|}{ Group } \\
\hline & \multicolumn{2}{|l|}{ Truth tellers } & \multicolumn{2}{|l|}{ Exaggerators } & \multicolumn{2}{|c|}{ Malingerers } \\
\hline$n$ & \multicolumn{2}{|l|}{64} & \multicolumn{2}{|l|}{63} & \multicolumn{2}{|l|}{40} \\
\hline Back or neck pain & \multicolumn{2}{|l|}{22} & \multicolumn{2}{|l|}{21} & \multicolumn{2}{|l|}{6} \\
\hline Headache (migraines) & \multicolumn{2}{|l|}{17} & \multicolumn{2}{|l|}{11} & \multicolumn{2}{|l|}{20} \\
\hline Ear or eye pain & \multicolumn{2}{|l|}{2} & \multicolumn{2}{|l|}{1} & \multicolumn{2}{|l|}{1} \\
\hline Joint pain or stiffness & \multicolumn{2}{|l|}{2} & \multicolumn{2}{|l|}{1} & & \\
\hline Chest pain & \multicolumn{2}{|l|}{1} & \multicolumn{2}{|l|}{1} & \multicolumn{2}{|l|}{2} \\
\hline Muscle pain & \multicolumn{2}{|l|}{2} & \multicolumn{2}{|l|}{8} & & \\
\hline Menstruation pain & \multicolumn{2}{|l|}{11} & \multicolumn{2}{|l|}{10} & \multicolumn{2}{|l|}{7} \\
\hline Abdominal pain & \multicolumn{2}{|l|}{3} & \multicolumn{2}{|l|}{7} & \multicolumn{2}{|l|}{3} \\
\hline Genital pain & \multicolumn{4}{|l|}{1} & \multicolumn{2}{|l|}{1} \\
\hline \multicolumn{7}{|l|}{ Added symptoms } \\
\hline Pain in leg/knee & \multicolumn{6}{|l|}{1} \\
\hline Physical injuries from soccer & \multicolumn{6}{|l|}{1} \\
\hline Sore throat & & & 1 & & & \\
\hline Toothache & 1 & & & & & \\
\hline Lower back/right hip & & & 1 & & & \\
\hline Tooth pain & & & 1 & & & \\
\hline Intensity & Initial & After instructions & Initial & After instructions & Initial & After instructions \\
\hline$M(S D)$ & $2.45(1.01)$ & $2.30(1.08)$ & $2.35(1.08)$ & $3.43(1.45)$ & / & $3.88(1.18)$ \\
\hline
\end{tabular}

exaggerators were asked to exaggerate symptoms, whereas participants in the malingering condition were asked to fabricate symptoms (instructions are provided in Appendix 1).

Following the instructions, all participants, regardless of the condition they were assigned to, were again asked to rate the intensity of their symptoms. This was done in order to check whether the participants complied with the instructions they were given. Consequently, the truth tellers and exaggerators rated their intensity twice and the malingerers only once. This is because the latter had not reported any genuine symptoms. All participants were then given two tasks: (1) To produce a narrative following their instructions. These statements were used in the application of the VA; and (2) to complete the SRSI. Finally, after completion of the questionnaire, participants were asked a couple of exit questions (e.g., "How motivated were you to fill out the questionnaire"), they were debriefed, and thanked for their cooperation. Participants were compensated either with a gift-voucher valued at 7.50 euros or one research participation credit. Partakers who were excluded due to the very high genuine intensity of symptoms they experienced were also rewarded with their choice of compensation.

\section{Statistical Analysis}

In order to investigate group differences, the following analyses of variances were carried out: (1) a multivariate analysis of variance (MANOVA) to assess differences between the groups (truth tellers, exaggerators, and malingerers) and their statement quality (i.e., number of verifiable details, number of non-verifiable details, proportion of verifiable information) as well as differences in their scores on the SRSI main scales (pseudosymptoms and genuine symptoms scale) and subscales; and (2) Bonferroni post hoc tests to follow-up the contrasts between each group on the variables mentioned above. Responses to exit questions were analyzed using a one-way analysis of variance (ANOVA) together with $t$ tests and post hoc tests (Bonferroni and Games-Howell) to assess other potential group differences (e.g., motivation, intensity of symptoms).

\section{Results}

\section{Exit Questions}

Using five-point scales (anchors, 1 = "Not at all", 5 = "A great deal") participants rated their motivation and the difficulty of the tasks.

Motivation We asked participants to rate their motivation for writing statements about their symptoms, filling out the questionnaire, and convincing us about the severity of their symptoms. The last one was only displayed to the exaggerators and malingerers groups. Overall, the whole sample reported moderate motivation to write a statement, $M=3.59, S D=.85$, and to fill out the SRSI, $M=3.59, S D=.84$. The three groups did not significantly differ in terms of motivation to write a 
statement and motivation to fill out the questionnaire, $F \mathrm{~s}(2$, $164)<2.30, p \mathrm{~s}>.103, \eta_{p}{ }^{2}<.027$, respectively. Exaggerators and malingerers together $(n=103)$ reported also moderate motivation to convince us in their complaint, $M=3.70$, $S D=.77$, and there was no statistically significant difference in their motivation, $t(101)=0.802, p=.425$.

Difficulty All participants were asked to rate the difficulty of providing a statement about symptoms and difficulty filling out the questionnaire. Both features were reported being somewhat easy with mean values $M=2.46(S D=1)$ and $M=2.12(S D=.87)$, respectively. While the difference on experienced difficulty writing a statement was not statistically significant between truth tellers, exaggerators, and malingerers, $F(2,164)=1.05, p=.354, \eta_{p}{ }^{2}=.013$, there was a statistically significant difference between groups in difficulty experienced while filling out the SRSI, $F(2,164)=8.25$, $p<.001, \eta_{p}{ }^{2}=.091$. A Bonferroni post hoc test revealed that the exaggerators rated the difficulty of filling out the questionnaire $(M=2.41, S D=.93)$ as significantly higher than truth tellers $(M=1.81, S D=.69 ; p<.001)$. Exaggerators and truth tellers did not differ significantly from malingerers $(M=2.15$, $S D=.89), p>.139$ and $p=.364$, respectively.

\section{Symptoms and Intensity Ratings}

Symptoms The most frequently reported symptoms were back or neck pain $(n=43,25.7 \%)$, followed by headaches/migraines $(n=28,16.8 \%)$ and menstruation pain $(n=21,12.6 \%)$. Forty participants reported not experiencing any kind of symptoms, and were asked to choose a symptom to fabricate. The most frequently fabricated symptoms corresponded to the prevalence of genuine complaints, just in a different order, headaches/ migraines $(n=20,50 \%)$, menstruation pain $(n=7,17.5 \%)$, and back or neck pain $(n=6,15 \%$; see Table 1$)$.

Intensity Ratings Participants graded the intensity of their symptoms on a 5-point Likert scale ( 1 = "very low" to $5=$ "extremely strong"). Truth tellers and exaggerators rated the intensity initially and after the instructions, whereas malingerers only rated after selecting fabricated symptom. The means are presented in Table 1. On initial report, truth tellers and exaggerators did not significantly differ, $t(125)=0.561, p=.576$.

A one-way ANOVA analysis on rated intensity after instructions showed that the three groups (i.e., truth tellers, exaggerators, and malingerers) differed significantly in their ratings, Welch's $F(2,97.68)=27.15, p<.001, \eta_{p}{ }^{2}=.22$. A follow-up analysis with a Games-Howell post hoc test (significant Levene's Test of Homogeneity of Variance, $p=.004$ ) revealed that the rated intensity was significantly lower for the truth tellers group compared with the exaggerators and malingerers. There was no statistically significant difference between the malingerers and exaggerators groups $(p=.206)$.
To check whether initial ratings of the truth teller and exaggerator groups differed significantly from ratings after instructions, a Paired samples $t$ test was performed. This analysis revealed that both truth tellers and exaggerators did significantly change their scores after the instructions, $t(63)=$ $-2.10, p=.04$, Cohen's $d=0.26$ and $t(62)=5.32, p<.001$, Cohen's $d=0.67$, respectively. However, the direction of these differences indicated that truth tellers significantly lowered their scores, whereas exaggerators reported significantly increased intensity the second time."

\section{Statement Quality}

The means and standard deviations on all of the statements' quality categories are presented in Table 2.

Length of Statements For the full sample $(N=167)$, the mean value of statement length was 148.56 words, $(S D=72.95)$, and it ranged between 36 and 623 words. Overall, there was a significant difference between groups in the length of the statements, $F(2,164)=5.46, p=.005, \eta_{p}{ }^{2}=.06$. Bonferroni post hoc test indicated that truth tellers provided significantly shorter statements than exaggerators, $p=.007$, and malingerers, $p=.05$. Exaggerators and Malingerers did not significantly differ in the length of their statements, $p=1.00$.

Verifiable, Non-Verifiable, and Proportion of Verifiable Details The average number of reported details (verifiable + non-verifiable) for the whole sample was $34.55(S D=15.36)$. Mean values for the sample regarding verifiable details and nonverifiable details were $M=5.08(S D=8.43)$ and $M=29.47$ $(S D=11.51)$, respectively. From the total number of reported details by the whole sample, only $14.70 \%$ were verifiable. Across the sample, 116 participants $(69.5 \%)$ reported at least one verifiable detail.

There was a significant difference between groups on the number of verifiable details, non-verifiable details, and the proportion of verifiable details, $F(6,324)=4.09, p=.001$, $\eta_{p}{ }^{2}=.070$. Simple effects were significant for the number of verifiable details, $F(2,164)=7.10, p=.001, \eta_{p}{ }^{2}=.080$, and for the proportion of verifiable details, $F(2,164)=9.12, p=<$ $.001, \eta_{p}{ }^{2}=.100$. However, groups did not significantly differ in terms of non-verifiable details, $F(2,164)=1.25, p=.289$, $\eta_{p}^{2}=.015$.

Post hoc tests showed that truth tellers provided significantly less verifiable information than exaggerators $(p=.002)$ and malingerers $(p=.017)$; no significant difference was found between exaggerators and malingerers $(p>.05)$. Considering the proportion of verifiable details, a similar pattern was found, with truth tellers' statements containing proportionally less checkable information than both exaggerators $(p<.001)$ and malingerers $(p=.041)$. The latter two groups did not differ significantly $(p>.05)$. 
Table 2 Means and standard deviations of statements' quality in truth tellers, exaggerators, and malingerers

\begin{tabular}{llll}
\hline Statement quality & Group & & \\
\cline { 2 - 4 } & Truth tellers $M(S D)$ & Exaggerators $M(S D)$ & Malingerers $M(S D)$ \\
\hline$n$ & 64 & 63 & 40 \\
Statement length & $125.58(55.11)$ & $164.32(85.79)$ & $160.53(68.03)$ \\
Verifiable details & $2.08(3.34)$ & $7.11(8.72)$ & $6.68(11.80)$ \\
Non-verifiable details & $27.81(10.95)$ & $29.98(12.61)$ & $31.33(10.44)$ \\
Proportion of verifiable details & $.06(.09)$ & $.17(.17)$ & $.14(.17)$ \\
Total details & $29.89(11.78)$ & $37.10(16.79)$ & $38.00(16.55)$ \\
\hline
\end{tabular}

\section{Symptom Endorsement on SRSI}

Table 3 presents the means and standard deviations of truth tellers, exaggerators, and malingerers on the main scales and subscales of the SRSI. Overall, there was a significant effect, $F(20,310)=1.82, p=.018, \eta_{p}{ }^{2}=.105$. The three groups significantly differed on the genuine symptom scale, $F(2,164)=$ $6.34, p=.002, \eta_{p}{ }^{2}=.072$, and pseudosymptoms scale, $F(2$, $164)=5.70, p=.004, \eta_{p}{ }^{2}=.065$. Post hoc tests showed that truth tellers endorsed significantly less genuine symptoms $(p=.002)$ and pseudosymptoms $(p=.003)$ than exaggerators but did not significantly differ from malingerers $(p=.133$ and $p=.514$, respectively). There was no significant difference between exaggerators and malingerers, neither in genuine symptoms $(p=.858)$ nor in pseudosymptoms scores $(p=.340)$.

Looking at the genuine subscales, the main differences between groups were found in the number of reported genuine cognitive, $F(2,164)=3.27, p=.04, \eta_{p}{ }^{2}=.04$, pain, $F(2$, $164)=12.86, p=.000, \eta_{p}{ }^{2}=.14$, and nonspecific somatic symptoms, $F(2,164)=5.71, p=.004, \eta_{p}{ }^{2}=.07$. Post hoc test revealed that, although the between subjects effect was significant, truth tellers, exaggerators, and malingerers did not significantly differ on the genuine cognitive symptoms subscale.
Truth tellers endorsed significantly less symptoms on the pain $(p<.001)$ and nonspecific somatic $(p=.004)$ symptoms than exaggerators, and significantly less pain symptoms than malingerers $(p=.008)$. There was no significant difference between exaggerators and malingerers on the SRSI genuine symptom subscales $(p s>.05)$.

Considering pseudosymptoms scales, differences were obtained on cognitive and pain subscales, $F(2,164)=3.24$, $p=.042, \eta p 2=.04$ and $F(2,164)=10.61, \mathrm{p}=.000$, $\eta \mathrm{p} 2=.12$, respectively. Post hoc comparisons showed that there was a significant difference exclusively between truth tellers and exaggerators. Specifically, truth tellers endorsed significant less cognitive/memory and pain pseudosymptoms compared to exaggerators $(p=.039$ and $p<.001$, respectively). Similar to the genuine symptom subscales, exaggerators and malingerers did not significantly differ on the pseudosymptom subscales $(p s>.05)$.

Detection Rate SRSI Previous research (Boskovic, Merckelbach, et al., 2019) proposed that the appropriate SRSI cutoff point for pain-related complaints is more than six pseudosymptoms endorsed. By employing this cutoff to our data, $47.6 \%$ of exaggerators $(n=30)$ and $40 \%$ of
Table 3 Means and standard deviations of SRSI main scales and subscales for truth tellers, exaggerators, and malingerers

\begin{tabular}{llll}
\hline SRSI & Truth Tellers $M(S D)$ & $\begin{array}{l}\text { Group } \\
\text { Exaggerators } M(S D)\end{array}$ & Malingerers $M(S D)$ \\
\hline$n$ & 64 & 63 & 40 \\
Genuine symptoms scale & $14.72(9.54)$ & $21.72(11.68)$ & $19.00(9.94)$ \\
Cognitive & $2.06(2.58)$ & $3.24(2.95)$ & $3.01(2.76)$ \\
Depression & $2.89(2.38)$ & $3.94(2.91)$ & $3.40(2.09)$ \\
Pain & $2.72(2.21)$ & $5.43(3.51)$ & $4.60(3.47)$ \\
Nonspecific somatic & $4.38(3.11)$ & $6.25(3.34)$ & $5.75(3.19)$ \\
PTSD/anxiety & $2.67(2.24)$ & $2.41(2.34)$ & $2.15(1.92)$ \\
Pseudosymptoms scale & $4.17(6.35)$ & $8.29(8.01)$ & $6.07(5.60)$ \\
Cognitive/memory & $0.92(1.81)$ & $1.71(1.87)$ & $1.45(1.57)$ \\
Neurological: motor & $0.55(1.48)$ & $1.05(1.64)$ & $0.50(0.85)$ \\
Neurological: sensory & $1.00(1.40)$ & $1.54(1.84)$ & $1.47(1.57)$ \\
Pain & $0.83(1.33)$ & $2.60(2.77)$ & $1.82(2.19)$ \\
Anxiety/depression/PTSD & $0.88(1.57)$ & $1.38(1.83)$ & $0.82(1.20)$ \\
\hline
\end{tabular}


malingerers $(n=16)$ were correctly classified as over-reporters. However, $20.3 \%$ of truth tellers $(n=13)$ were wrongly classified as over-reporters (i.e., false positives; see Table 4).

\section{Discussion}

The current study has examined whether applying two recent detection approaches, the VA and the SRSI, could aid the differentiation between truth tellers, exaggerators, and malingerers.

The main study outcomes can be summarized as follows: (i) The most frequently reported genuine complaints were back or neck pain, headaches, and menstruation pain. The last is not surprising as most of our participants were women. These finding fits nicely with previous research showing a high prevalence of chronic pain-related issues among student population (Hadjistavropoulos et al., 2007). Moreover, the same symptoms were also the most frequently chosen by malingerers, which is in accordance with previous findings that students who are willing to malinger most often opt for headaches, back pain, and menstruation pain (Boskovic, 2019a). (ii) Truth tellers provided shorter statements than both exaggerators and malingerers. There was no statistically significant difference in statements' length between exaggerators and malingerers. This finding confirms previous results of the VA in symptom validity context (Boskovic et al., 2017; Boskovic et al., 2018; Boskovic, Dibbets, et al., 2019). Fabricators of symptoms (i.e., exaggerators and malingerers in this study), were shown to be inclined to enrich their statements with more details so as to manipulate their veracity (Akehurst et al., 2017; Boskovic et al., 2017; Boskovic, Dibbets, et al., 2019; Nahari \& Nisin, 2019). Of importance to this study is that exaggerators and malingerers, when compared with each other, reported a similar quantity of details in their reports, without any significant differences between them. This lack of differences suggests that longer reports are indeed a general cue to deception in symptom validity context, however, it lacks sensitivity to different levels of symptom deceitfulness.

(iii) As previous studies showed (e.g., Boskovic et al., 2017; Boskovic et al., 2018), fabricators report more detailed

Table 4 Detection rate of SRSI in truth tellers, exaggerators, and malingerers

\begin{tabular}{lll}
\hline Group & SRSI pass $(\leq 6)$ & SRSI fail $(\geq 6)$ \\
\hline Truth tellers & $51(79.7 \%)$ & $13(20.3 \%)$ \\
Exaggerators & $33(52.4 \%)$ & $30(47.6 \%)$ \\
Malingerers & $24(60 \%)$ & $16(40 \%)$ \\
\hline
\end{tabular}

Note: "fail" implies over-reporting tendency, "pass" is indicative of normal symptom endorsement propensity statements than people with genuine symptoms, usually using more vague, non-verifiable information. However, contrary to our expectations and previous findings, our results showed no significant differences between truth tellers, exaggerators, and malingerers in terms of non-verifiable details. Rather, the difference between groups emerged in the number and proportion of verifiable details. Specifically, truth tellers reported a significantly lower proportion of verifiable information than exaggerators and malingerers, explaining the differences in number of total details and in the length of statements reported above. Thus, our findings contradict the original assumption of the VA that argues that the proportion of verifiable details is a stable indicator of truthfulness (Nahari, 2018; Vrij, 2016). However, this discrepancy in findings might be specifically related to the subjective nature of pain symptoms. Previous research on the VA in symptom validity context also showed a lack of utility for the proportion of verifiable details in detecting malingering (Boskovic et al., 2018), but it did provide support for the use of non-verifiable information. Yet, it may be that the subjective descriptions (non-verifiable information) do not aid differentiation between people with genuine symptoms and those who fabricate them, specifically in pain-related reports. Rather, the abundance of verifiable information (e.g., prescription, witnesses), which is usually lacking in these types of symptom reports, appears to indicate premeditation, which is present in those who intentionally exaggerate/fabricate symptoms. The latter might be a more plausible explanation considering previous research showing strong tendencies among malingerers to produce false verifiable details by including false witnesses (e.g., parents) in their statements (Boskovic et al., 2017).

(iv) Considering the SRSI results, truth tellers reported fewer symptoms on both main SRSI scales; however, the differences only reached significance when compared with exaggerators. Thus, no differences were found between truth tellers and malingerers, nor exaggerators and malingerers in terms of genuine symptoms and pseudosymptoms. These findings are unusual, considering repeated findings that support malingerers' "over-the-top" strategy of endorsing symptoms (Boskovic, Merckelbach, et al., 2019; Merten et al., 2016). In this study, however, it was shown that, although both exaggerators and malingerers exhibited over-reporting, this tendency was slightly stronger among exaggerators, who had an underlying genuine condition that led to a significant difference only between exaggerators and truth tellers.

(v) Previous studies showed that the SRSI has limited utility in detecting fabricated physical complaints, reporting a detection rate of only $42 \%$ (Boskovic, Merckelbach, et al., 2019). These previous findings fit well with our results that showed that the highest detection rate reached $47.6 \%$ among exaggerators, whereas among malingerers it dropped to $40 \%$. Unfortunately, $20 \%$ of truth tellers were also detected, indicating a high level of false positives. Taken together, these results 
signal an issue in SRSI's applicability in the field of painrelated complaints. In addition, the lack of differences between the truth tellers and malingerers, as well as between exaggerators and malingerers, especially on pseudosymptoms scales, flags the limited sensitivity of the SRSI in differentiating genuine, exaggerated, and fully fabricated pain-related health complaints.

Certain limitations in this study need to be addressed. First, the generalizability of our findings is limited. Our sample consisted of students, and their symptom reports in this simulation study might not imitate reports genuine patients' accounts. Further, due to ethical restrictions, we did not include participants who surpassed a threshold of experienced symptom intensity, which possibly induced important differences between the current sample and an actual patient sample. Second, our study relied on participants' self-report which led to an inability to know whether the disclosed physical complaints were genuine (see Kucyi, Scheinman, \& Defrin, 2015). However, the subjective characteristic of pain does not allow for any other way of testing. Third, our study was conducted online using Qualtrics, and this meant that we were not able to personally ensure that every participant was focused and compliant with the instructions. Research has shown that a large number of online participants respond to questions unattentively (Fleischer, Mead, \& Huang, 2015). To combat this issue, we employed checks such as filler items in the SRSI and manipulation checks such as repeated intensity questions. The findings that truth tellers reported lower symptom intensity the second time, which might suggest presence of subject-expectancy effect (Supino, 2012). Our results showed that participants passed the checks for random responding, and that they were compliant with the instructions. Finally, concerns exist regarding the design employed in this study. We used the simulation design which instructs partakers to simulate real-world settings by using scenarios (Rogers, 2018b). Hence, the ecological validity of our findings is limited.

Overall, our findings indicate limited utility of both the SRSI and VA in differentiating genuine, exaggerated, and malingered pain reports. Our main findings contradict previous research using these two methods (e.g., Boskovic et al., 2017; Merten et al., 2016). The differences captured by the SRSI in this study suggest that an over-reporting tendency, often cited as the trademark of pure malingering (Merten et al., 2016; Boskovic, 2019b), may actually be more intense among individuals who have genuine pain symptoms but exaggerate them. By inspecting the verbal quality of accounts, we confirmed previous findings that deceivers put more effort in writing rich narratives, making them significantly longer than genuine symptom reports (Boskovic et al., 2017). However, we did not find support for verifiable information being indicative of veracity, rather we suggest that it may signal symptom deception in pain-related reports. Yet, due to the inconsistent findings, future research, preferably with patients, is necessary in order to establish the divergent capability of symptom validity methods in distinguishing exaggerators and malingerers.

Availability of Data and Material Data is available at Open Science Framework (OSF) platform https://osf.io/9d4mp/.

Authors' Contributions First author collected the data, coded the statements, and drafted the manuscript. Second author helped coding the data and revising the manuscript. Last author was the creator and the supervisor of the project, and had took part in all steps of the project.

Funding Information We did not receive any external funding for this project.

\section{Compliance with Ethical Standards}

Conflicts of Interest The authors declare that they have no conflict of interest.

\section{Appendix 1}

\section{Group Instructions}

\section{Truth Tellers}

Dear participant,

You reported currently suffering from a certain pain symptom. In this study, we want you to focus on your sensations and activities you usually take when you are experiencing pain. Precisely, we will ask you to write about one typical day in which you experience your symptoms. Please write a detail statement about that day, including both the sensations (e.g., burning and cramps) and the activities (e.g., talking to friends, taking medication) you take in order to cope with the symptoms. After the statement, you will be given the questionnaire to which we also ask you to honestly respond. Please perform the tasks in an honest manner. At the end of the study, you will be presented with a few exit questions. Thank you for your participation!

\section{Exaggerators}

Dear participant,

You reported currently suffering from a certain pain symptom. In this study, we want you to focus on your sensations and activities you usually take when you are experiencing pain and exaggerate them. Precisely, we will ask you to imagine that you missed the final resit. You did have your current symptom on that day, but you were still able to attend the exam. However, you overslept and missed it. Your only option to have one more 
chance to take this exam is to exaggerate your symptoms and submit the report to the exam committee that will convince them that there was no possibility of you attending the resit due to the excruciating pain that you experienced. Now, imagine that we are the exam committee and that through the next questions and tasks you need to convince us that your pain is much greater than what it is. We will first ask you to:

1) Write a report about the day of the resit. Please write a detailed statement about that day, including both the sensations (e.g., burning and cramps) and the activities (e.g., talking to friends, taking medication) you took in order to cope with the symptoms.

2) After the statement, you will be given the questionnaire to which we also ask you to respond keeping in mind the situation of the resit. Remember that if you write a convincing report and convincingly fill out the questionnaire, you will be allowed to have another resit.

Please keep this instruction in mind while answering the questions and performing the tasks on next pages! If you provide a convincing report, you will be entered a raffle in which you can earn a bonus of $€ 10$ ! At the end of the study, you will be presented with a few exit questions. Thank you for your participation!

\section{Malingerers}

\section{Dear participant,}

You reported not currently suffering from any pain symptoms. However, in this study, we want you to imagine as if you currently have a pain-related symptom, and we ask you to focus on your sensations and activities you would usually take when you are experiencing pain. Precisely, we will ask you to imagine that you missed the final resit. You did not have any health issues on that day, however, you overslept and missed it. Your only option to have one more chance to take this exam is to fabricate suffering from a severe pain and submit the report to the exam committee that will convince them that there was no possibility of you attending the resit due to the excruciating pain that you experienced. Now, imagine that we are the exam committee and that through the next two tasks you need to convince us that your pain is real and severe. We will first ask you to:

1) Tell us which symptom you suffer from (choose one pain-related symptoms from the list that you will report (falsely) about) and report its intensity.

2) Write a report about the day of the resit. Please write a detailed statement about that day, including both the sensations (e.g., burning and cramps) and the activities (e.g., talking to friends, taking medication) you took in order to cope with the symptoms.
3) After the statement, you will be given the questionnaire to which we also ask you to respond keeping in mind the situation of the resit. Remember that if you write a convincing report and convincingly fill out the questionnaire, you will be allowed to have another resit. Please keep this instruction in mind while answering the questions and performing the tasks on next pages! If you provide a convincing report, you will be entered into a raffle in which you can earn a bonus of $€ 10$ ! At the end of the study, you will be presented with a few exit questions. Thank you for your participation!

Open Access This article is licensed under a Creative Commons Attribution 4.0 International License, which permits use, sharing, adaptation, distribution and reproduction in any medium or format, as long as you give appropriate credit to the original author(s) and the source, provide a link to the Creative Commons licence, and indicate if changes were made. The images or other third party material in this article are included in the article's Creative Commons licence, unless indicated otherwise in a credit line to the material. If material is not included in the article's Creative Commons licence and your intended use is not permitted by statutory regulation or exceeds the permitted use, you will need to obtain permission directly from the copyright holder. To view a copy of this licence, visit http://creativecommons.org/licenses/by/4.0/.

\section{References}

Akehurst, L., Easton, S., Fuller, E., Drane, G., Kuzmin, K., \& Litchfield, S. (2017). An evaluation of a new tool to aid judgements of credibility in the medico-legal setting. Legal and Criminological Psychology, 22, 22-46. https://doi.org/10.1111/lcrp.12079.

American Psychiatric Association. (2013). Diagnostic and statistical manual of mental disorders (5th ed.). Washington, DC: Author.

Bender, S. D., \& Matusewicz, M. (2013). PCS, iatrogenic symptoms, and malingering following concussion. Psychological Injury and Law, 6, 113-121. https://doi.org/10.1007/s12207-013-9156-9.

Boskovic, I. (2019a). Do motives matter? A comparison between positive and negative incentives in students' willingness to malinger. Educational Psychology, 1-11. https://doi.org/10.1080/01443410. 2019.1704400.

Boskovic, I. (2019b). A multi-method approach to the detection of fabricated symptoms. Doctoral dissertation. Maastricht University, Maastricht. Retrieved from https://doi.org/10.26481/dis.20190129ib

Boskovic, I., Bogaard, G., Merckelbach, H., Vrij, A., \& Hope, L. (2017). The verifiability approach to detection of malingered physical symptoms. Psychology, Crime \& Law, 23, 717-729. https://doi.org/10. 1080/1068316x.2017.1302585.

Boskovic, I., Dibbets, P., Bogaard, G., Hope, L., Jelicic, M., \& Orthey, R. (2019). Verify the scene, report the symptoms: Testing the verifiability approach and SRSI in the detection of fabricated PTSD claims. Legal and Criminological Psychology., 24, 241-257. https://doi. org/10.1111/lcrp.12149.

Boskovic, I., Gallardo, C. T., Vrij, A., Hope, L., \& Merckelbach, H. (2018). Verifiability on the run: An experimental study on the verifiability approach to malingered symptoms. Psychiatry, Psychology and Law, 26, 1-12. https://doi.org/10.1080/13218719.2018.1483272.

Boskovic, I., Merckelbach, H., Merten, T., Hope, L., \& Jelicic, M. (2019). The self-report symptom inventory as an instrument for detecting symptom overreporting: An exploratory study with instructed 
simulators. European Journal of Psychological Assessment., 1-10. https://doi.org/10.1027/1015-5759/a000547.

Fleischer, A., Mead, A. D., \& Huang, J. (2015). Inattentive responding in MTurk and other online samples. Industrial and Organizational Psychology, 8, 196-202. https://doi.org/10.1017/iop.2015.25.

Giger, P., \& Merten, T. (2019). Equivalence of the German and the French versions of the self-report symptom inventory. Swiss Journal of Psychology, 78, 5-13. https://doi.org/10.1024/1421-0185/a000218.

Hadjistavropoulos, H., Dash, H., Hadjistavropoulos, T., \& Sullivan, T. (2007). Recurrent pain among university students: Contributions of self-efficacy and perfectionism to the pain experience. Personality and Individual Differences, 42, 1082-1091.

Harzing, A. W. (2006). Response styles in cross-national survey research: A 26-country study. International Journal of Cross Cultural Management, 6(2), 243-266. https://doi.org/10.1177/ 1470595806066332.

Iverson, G. L. (2006). Ethical issues associated with the assessment of exaggeration, poor effort, and malingering. Applied Neuropsychology, 13, 77-90. https://doi.org/10.1207/ s15324826an1302_3.

Iverson, G. L. (2007). Identifying exaggeration and malingering. Pain Practice, 7, 94-102. https://doi.org/10.1111/j.1533-2500.2007.00116.x.

Koo, T. K., \& Li, M. Y. (2016). A guideline of selecting and reporting intraclass correlation coefficients for reliability research. Journal of Chiropractic Medicine, 15, 155-163. https://doi.org/10.1016/j.jcm. 2016.02.012.

Kucyi, A., Scheinman, A., \& Defrin, R. (2015). Distinguishing feigned from sincere performance in psychophysical pain testing. The Journal of Pain, 16, 1044-1053. https://doi.org/10.1016/j.jpain.2015.07.004.

McCracken, L. M. (2005). Social context and acceptance of chronic pain: The role of solicitous and punishing responses. Pain, 113(1-2), $155-159$.

Merckelbach, H., Boskovic, I., Pesy, D., Dalsklev, M., \& Lynn, S. J. (2017). Symptom overreporting and dissociative experiences: A qualitative review. Consciousness and Cognition, 49, 132-144. https://doi.org/10.1016/j.concog.2017.01.007.

Merten, T., Giger, P., Merckelbach, H., \& Stevens, A. (2019). SRSI: SelfReport Symptom Inventory-deutsche Version. Hogrefe, Germany.

Merten, T., \& Merckelbach, H. (2013). Symptom validity testing in somatoform and dissociative disorders: A critical review. Psychological Injury and Law, 6, 122-137. https://doi.org/10. 1007/s 12207-013-9155-x.

Merten, T., Merckelbach, H., Giger, P., \& Stevens, A. (2016). The selfreport symptom inventory (SRSI): A new instrument for the assessment of distorted symptom endorsement. Psychological Injury and Law, 9, 102-111. https://doi.org/10.1007/s12207-016-9257-3.

Mittenberg, W., Patton, C., Canyock, E. M., \& Condit, D. C. (2002). Base rates of malingering and symptom exaggeration. Journal of Clinical and Experimental Neuropsychology, 24, 1094-1102. https://doi.org/ 10.1076/jcen.24.8.1094.8379.

Nahari, G. (2018). Chapter 14 - the applicability of the verifiability approach to the real world. In J. P. Rosenfeld (Ed.), Detecting concealed information and deception (pp. 329-349): Academic press.

Nahari, G., \& Nisin, Z. (2019). Digging further into the speech of liars: Future research prospects in verbal lie detection. Frontiers in Psychiatry, 10, 56.

Nahari, G., \& Vrij, A. (2014). Can I borrow your alibi? The applicability of the verifiability approach to the case of an alibi witness. Journal of Applied Research in Memory and Cognition, 3, 89-94. https://doi. org/10.1016/j.jarmac.2014.04.005.

Nahari, G., Vrij, A., \& Fisher, R. P. (2014). Exploiting liars' verbal strategies by examining the verifiability of details. Legal and
Criminological Psychology, 19, 227-239. https://doi.org/10.1111/j. 2044-8333.2012.02069.x.

Nelson, N. W., Hoelzle, J. B., Sweet, J. J., Arbisi, P. A., \& Demakis, G. J. (2010). Updated meta-analysis of the MMPI-2 symptom validity scale (FBS): Verified utility in forensic practice. The Clinical Neuropsychologist, 24(4), 701-724. https://doi.org/10.1080/ 13854040903482863.

Nicholson, K. (2000). At the crossroads: Pain in the 21 st century. NeuroRehabilitation, 14, 57-67. https://doi.org/10.3233/NRE2000-14202.

Nicholson, K., \& Martelli, M. (2007). Malingering: Chronic pain. In G. Young, A. Kane, \& K. Nicholson (Eds.), Causality of psychological injury: Presenting evidence in court (pp. 477-500). New York, NY: Springer.

Nijdam-Jones, A., \& Rosenfeld, B. (2017). Cross-cultural feigning assessment: A systematic review of feigning instruments used with linguistically, ethnically, and culturally diverse samples. Psychological Assessment, 29, 1321-1336. https://doi.org/10. 1037/pas0000438.

Peace, K. A., \& Bouvier, K. A. (2008). Alexithymia, dissociation, and social desirability: Investigating individual differences in the narrative content of false allegations of trauma. Journal of Offender Rehabilitation, 47(1-2), 138-167. https://doi.org/10.1080/ 10509670801941035.

Peace, K. A., \& Masliuk, K. A. (2011). Do motivations for malingering matter? Symptoms of malingered PTSD as a function of motivation and trauma type. Psychological Injury and Law, 4, 44-55. https:// doi.org/10.1007/s12207-011-9102-7.

Petrie, K. J., Faasse, K., Crichton, F., \& Grey, A. (2014). How common are symptoms? Evidence from a New Zealand national telephone survey. BMJ Open, 4, e005374. https://doi.org/10.1136/bmjopen2014-005374.

Resnick, P. J. (1984). The detection of malingered mental illness. Behavioral Sciences \& the Law, 2, 21-38. https://doi.org/10.1002/ bsl.2370020104.

Resnick, P. J., West, S. G., \& Wooley, C. N. (2018). The malingering of posttraumatic disorders. In R. Rogers \& S. D. Bender (Eds.), Clinical assessment of malingering and deception (4th ed., pp. 188-211). New York, NY: Guilford Press.

Rogers, R. (2018a). An introduction to response styles. In R. Rogers \& S. D. Bender (Eds.), Clinical assessment of malingering and deception (4th ed., p. 10). New York, NY: Guilford Press.

Rogers, R. (2018b). Researching response styles. In R. Rogers \& S. D. Bender (Eds.), Clinical assessment of malingering and deception (pp. 592-614). New Yoirk, NY: Guilford Press.

Supino, P. G. (2012). Fundamental issues in evaluating the impact of interventions: Sources and control of bias. In Principles of Research Methodology (pp. 79-110). Springer, New York, NY. https://doi.org/10.1007/978-1-4614-3360-6 5.

van Helvoort, D., Merckelbach, H., \& Merten, T. (2019). The self-report symptom inventory (SRSI) is sensitive to instructed feigning, but not to genuine psychopathology in male forensic inpatients: An initial study. The Clinical Neuropsychologist, 33, 1069-1082. https://doi.org/10.1080/13854046.2018.1559359.

Vrij, A. (2016). Baselining as a lie detection method. Applied Cognitive Psychology, 30(6), 1112-1119. https://doi.org/10.1002/acp.3288.

Young, G. (2015). Malingering in forensic disability-related assessments: Prevalence $15 \pm 15 \%$. Psychological Injury and Law, 8, 188-199. https://doi.org/10.1007/s12207-015-9232-4.

Publisher's Note Springer Nature remains neutral with regard to jurisdictional claims in published maps and institutional affiliations. 